\title{
PID control of FOPDT plants with dominant dead time based on the modulus optimum criterion
}

\begin{abstract}
JAN CVEJN
The modulus optimum (MO) criterion can be used for analytical design of the PID controller for linear systems with dominant dead time. However, although the method usually gives fast and non-oscillating closed-loop responses, in the case of large dead time the stability margin gets reduced and even non-stable behavior can be observed. In this case a correction of the settings is needed to preserve the stability margin. We describe and compare two methods of design of the PID controller based on the MO criterion that for the stable first-order systems with dead time preserve the stability margin, trying to keep maximum of the performance of the original MO settings.
\end{abstract}

Key words: PID controller, process control, dead time, modulus optimum, magnitude optimum.

\section{Introduction}

The PID controller is a basic control instrument, which has been used in a majority of control applications in industry [1]. Although this structure of a controller has been widely utilized since the first half of 20th century, the problem of designing the PID controller by means of its three tuning parameters is still alive and topical. This fact is documented by the number of scientific papers on this problem, which appeared in the last decades. This is true even in the case of control of linear time-invariant systems, because the requirements on the closed-loop stability and robustness create complicated constraints for the design objective [2].

This paper is focused on the problem of tuning the PID controller for the systems with transfer function in Laplace transform

$$
F(s)=\frac{K}{T s+1} e^{-\tau s}
$$

where $K$ is the system gain, $T>0$ is the time constant and $\tau>0$ the dead-time parameter. $\tau$ is considered sufficiently large with respect to $T$, so the dead-time dynamics is

J. Cvejn is with Faculty of Electrical Engineering and Informatics, University of Pardubice, Studentska 95, 532 10, Pardubice, Czech Republic. E-mail: jan.cvejn@upce.cz

Received 22.04.2015. Revised 29.10.2015. 
important for the plant behavior. This simple plant is often being used for approximate modeling of processes with non-oscillating high-order dynamics and allows simple experimental identification from the step response, which can be in most cases easily measured. Simple methods based on coincidence in one or more points and more complex methods suitable for noisy data are described e.g. in [1] and [3].

For tuning the PID controller based on the model (1) many approaches exist, see e.g. [1] for a description of the most important methods. A comprehensive survey of known formulas is available in [4]. Early methods were derived from empirical requirements on the step response, such as one-quarter decay ratio [5], [6], step-response overshoot [7] or from integral criterions in time domain with approximation of the dead-time dynamics [8]. These methods, however, usually work well only for a rather limited range of the ratio $\tau / T$.

The design based on the well-known modulus optimum (MO) criterion [9], [1], [10], [14] is one of the approaches that allow working even with long $\tau$ with respect to $T$. This design criterion requires that the closed loop frequency response modulus is as flat as possible in the range of low frequencies, i.e.

$$
\lim _{\omega \rightarrow 0}\left|\frac{L(i \omega)}{1+L(i \omega)}\right|=1, \quad \lim _{\omega \rightarrow 0} \frac{d^{k}}{d \omega^{k}}\left|\frac{L(i \omega)}{1+L(i \omega)}\right|=0, \quad k=1,2, \ldots, k_{m}
$$

where $L(i \omega)$ denotes the open-loop frequency response, i.e. the product of the responses of the plant and the controller, and $k_{m}$ is as high as possible. The MO-based design is most natural for the reference tracking control tasks, where the closed-loop system is to be able to respond quickly to changes of the reference input, or equivalently to efficiently reject the disturbances influencing directly the plant output. In this case it usually produces fast non-oscillating responses [10], [14].

The PID controller can be for the plant (1) designed in a simplified way if the factor $(T s+1)$ in (1) is compensated by the controller zero. Then only the two remaining control loop parameters are to be determined with respect to the MO criterion. This approach, utilized in [13], has several important advantages: it preserves a sufficient stability margin, gives simple tuning rules and it enables to propose a compensation of the settings in the cases when the disturbance influences the plant input. The resulting MO-based tuning rules

$$
K_{c}=\frac{3}{4} \frac{T}{K \tau}, \quad T_{s I}=T, \quad T_{s d}=\frac{\tau}{3}
$$

where the PID controller is considered in the serial form

$$
R(s)=K_{c}\left(1+\frac{1}{T_{s I} s}\right)\left(1+T_{s d} s\right)
$$

offer fast and well damped responses even for large $\tau / T$, unlike most other tuning methods. For the parallel PID controller

$$
R(s)=K_{c}\left(1+\frac{1}{T_{I} s}+T_{d} s\right)
$$


the settings corresponding to (3) can be rewritten as

$$
K_{c}=\frac{1}{4 K}\left(1+\frac{3 T}{\tau}\right), \quad T_{I}=T+\frac{\tau}{3}, \quad T_{d}=\frac{\tau}{3+\tau / T}
$$

In this paper the design of the PID controller for the plant (1) is considered in full form, where all the three controller parameters are determined with respect to the MO criterion and no pole/zero compensation is used. In comparison with the simplified approach mentioned above it can be assumed that the performance is enhanced in the sense that the closed-loop magnitude response is more flat in the origin, which means that the bandwidth is increased. On the other hand, we show that if the ratio $\tau / T$ is sufficiently large, even non-stable closed loop can be obtained in this way. Therefore, a modification of the settings is needed to preserve the stability margin. This problem has been treated already in [12], where a simple correction of the MO settings was proposed.

In [11] the problem of the MO design is analyzed for the more general plant

$$
F(s)=\frac{K}{\left(T_{1} s+1\right)\left(T_{2} s+1\right) \cdots\left(T_{n} s+1\right)} e^{-\tau s}
$$

where $T_{1} \geqslant T_{2} \geqslant \cdots \geqslant T_{n}>0$ and $\tau>0$. It was observed that the MO-optimal controller parameters are positive and the corresponding open-loop Nyquist plot for the plants (7) lies in the half-plane $\{z \mid \operatorname{Re} z \geqslant-0.5\}$ if $\tau$ is not too large. This property ensures a large stability margin and good robustness of the MO settings, although in the cases of long dead time a correction of the MO settings is needed. The correction of the settings proposed in [11] is designed to preserve maximum of the performance, which in a certain sense corresponds to the flatness of the closed-loop magnitude response for low frequencies. However, the algorithm of this correction is iterative, although the computation is simple and very efficient.

Based on the results in [11], in this paper we propose a modification of the MObased PID controller settings for the FOPDT plant (1), which guarantee stability and the stability margin specified above for any $\tau>0$ and are fully explicit, i.e. can be obtained without any iterative computations.

\section{The MO tuning of PID controller}

If we rewrite the transfer function (7) as

$$
F(s)=\frac{K}{A_{n} s^{n}+\cdots+A_{1} s+1} e^{-\tau s}
$$

the corresponding MO-optimal PID controller (5) settings for the system (7) are in [11] obtained in the form

$$
K_{c}=\frac{r_{0}}{K}, \quad T_{I}=\frac{r_{0} \tau}{r_{-1}}, \quad T_{d}=\frac{r_{1} \tau}{r_{0}}
$$


where the parameters $r_{i}, i=-1,0,1$ are obtained as the solution to the following system of linear equations:

$$
\left(\begin{array}{ccc}
\sum_{j=0}^{1} \frac{a_{1-j}}{j !} & -1 & 0 \\
\sum_{j=0}^{3} \frac{a_{3-j}}{j !} & -\sum_{j=0}^{2} \frac{a_{2-j}}{j !} & \sum_{j=0}^{1} \frac{a_{1-j}}{j !} \\
\sum_{j=0}^{5} \frac{a_{5-j}}{j !} & -\sum_{j=0}^{4} \frac{a_{4-j}}{j !} & \sum_{j=0}^{3} \frac{a_{3-j}}{j !}
\end{array}\right)\left(\begin{array}{c}
r_{-1} \\
r_{0} \\
r_{1}
\end{array}\right)=\frac{1}{2}\left(\begin{array}{c}
1 \\
2 a_{2}-a_{1}^{2} \\
2 a_{4}+a_{2}^{2}-2 a_{1} a_{3}
\end{array}\right)
$$

where

$$
a_{j}=\frac{A_{j}}{\tau^{j}}, \quad a_{0}=1 .
$$

For the plant (1) $a_{1}=T / \tau$ and $a_{2}=a_{3}=\cdots=0$ in (10), so we obtain

$$
\left(\begin{array}{ccc}
\eta+1 & -1 & 0 \\
\frac{\eta}{2}+\frac{1}{6} & -\left(\eta+\frac{1}{2}\right) & \eta+1 \\
\frac{\eta}{4 !}+\frac{1}{5 !} & -\left(\frac{\eta}{6}+\frac{1}{4 !}\right) & \frac{\eta}{2}+\frac{1}{6}
\end{array}\right)\left(\begin{array}{l}
r_{-1} \\
r_{0} \\
r_{1}
\end{array}\right)=\frac{1}{2}\left(\begin{array}{c}
1 \\
-\eta^{2} \\
0
\end{array}\right)
$$

where $\eta=T / \tau$, which can be rewritten as follows:

$$
\left(\begin{array}{ccc}
\eta+1 & -1 & 0 \\
3 \eta+1 & -3(2 \eta+1) & 6(\eta+1) \\
5 \eta+1 & -5(4 \eta+1) & 20(3 \eta+1)
\end{array}\right)\left(\begin{array}{l}
r_{-1} \\
r_{0} \\
r_{1}
\end{array}\right)=\left(\begin{array}{c}
0.5 \\
-3 \eta^{2} \\
0
\end{array}\right)
$$

The solution to (13) can be expressed in the explicit form:

$$
\begin{gathered}
r_{0}=\frac{1}{D}\left(180 \eta^{4}+240 \eta^{3}+135 \eta^{2}+42 \eta+7\right) \\
r_{1}=\frac{1}{D}\left(60 \eta^{4}+60 \eta^{3}+27 \eta^{2}+7 \eta+1\right) \\
r_{-1}=\frac{15}{D}\left(12 \eta^{3}+12 \eta^{2}+5 \eta+1\right)
\end{gathered}
$$

where

$$
D=16\left(15 \eta^{3}+15 \eta^{2}+6 \eta+1\right)
$$

Note that the settings exist for any $\eta>0$. The actual PID controller parameters are obtained by substitution into (9).

Fig. 1 shows the open-loop Nyquist plots for the plants (1), for three values of $T$ and $\tau=1$. Fig. 2 shows the corresponding closed-loop step responses. In the simulations there is considered the $1 s t$-order low-pass filter with time constant $T_{f}=0.02$ [sec] by 
the derivative term of the controller (not considered at the controller design stage). This means, the actual PID controller transfer function is

$$
R(s)=K_{c}\left(1+\frac{1}{T_{I} s}+\frac{T_{d} s}{0.02 s+1}\right) .
$$

The same modification of the controller, corresponding to a real situation, was used for obtaining the other simulated results in this paper as well (Figs 4 and 6).

It can be seen from Fig. 1 that the optimal open-loop Nyquist plot is flat and curving towards the origin for low frequencies. Note that the performance gets enhanced with decreasing $T$ in the sense that the closed-loop magnitude response is more flat in the origin. On the other hand, for sufficiently low $T$ the open-loop magnitude increases for higher frequencies, which has ill effect on the stability margin, although it is true that this phenomenon can be observed only for rather large values of $\tau / T$. In the time domain this problem corresponds to undesirable oscillations in the step response

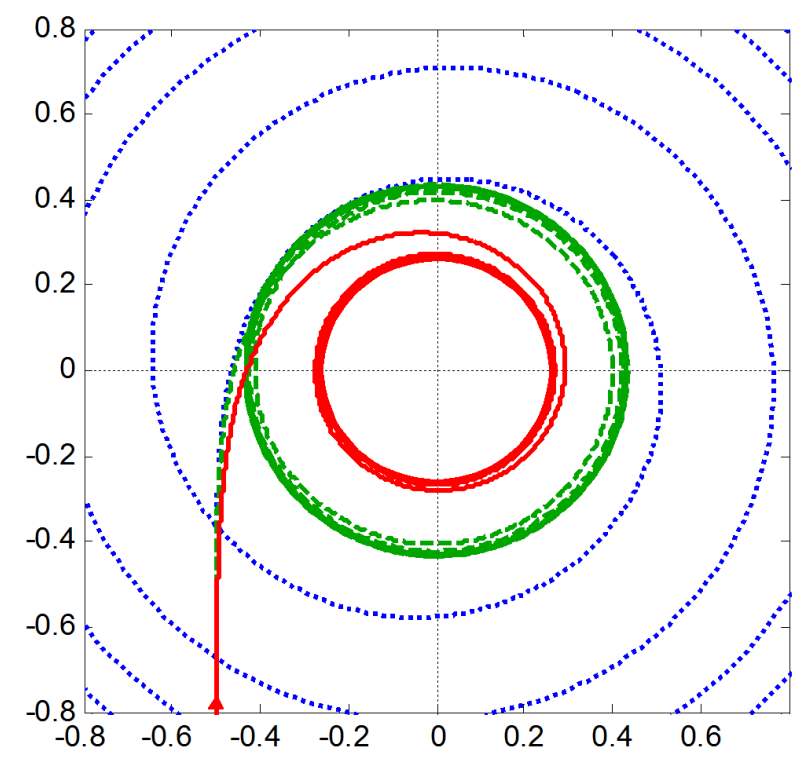

Figure 1. The MO-optimal open-loop Nyquist plots for plants (1) where $\tau=1$ [sec] and $T=1$ [sec] (solid line), $T=0.2[\mathrm{sec}]$ (dashed line) and $T=0.05$ [sec] (dotted line). 


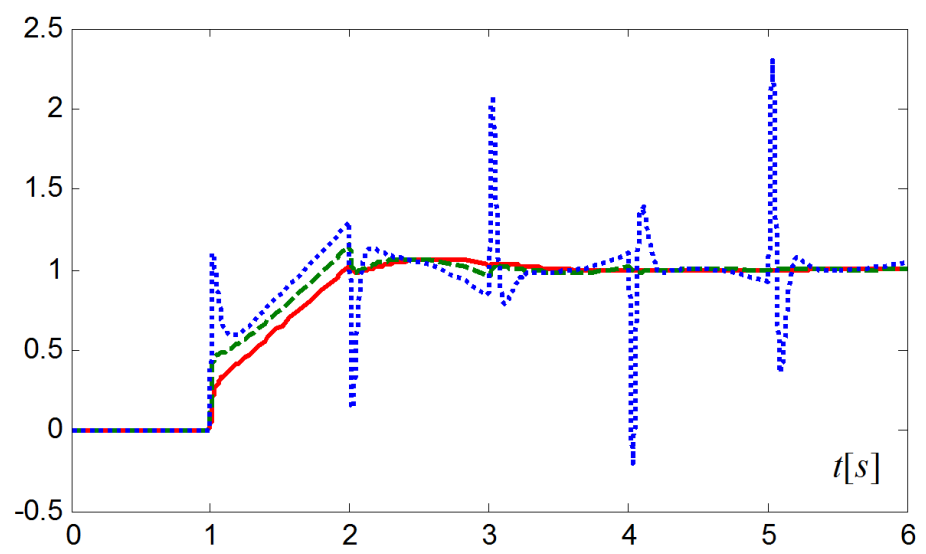

Figure 2. The MO-optimal step responses for plants (1) where $\tau=1$ [sec] and $T=1$ [sec] (solid line), $T=0.2[\mathrm{sec}]$ (dashed line) and $T=0.05[\mathrm{sec}]$ (dotted line).

\section{The correction of the MO settings}

\subsection{The simplified approach}

If we use (9), the open-loop transfer function can be written as

$$
\begin{aligned}
R(s) F(s) & =K_{c}\left(1+\frac{1}{T_{I} s}+T_{d} s\right) \frac{K}{T s+1} e^{-\tau s}= \\
& =\frac{r_{0}}{K}\left(1+\frac{r_{-1}}{r_{0} \tau} \frac{1}{s}+\frac{r_{1} \tau}{r_{0}} s\right) \frac{K}{T s+1} e^{-\tau s}=\left(r_{0}+r_{-1} \frac{1}{\tau s}+r_{1} \tau s\right) \frac{e^{-\tau s}}{T s+1} .
\end{aligned}
$$

If we put $\xi=\tau \omega$, the open-loop frequency response, corresponding to (19), can be written in the form

$$
L(\xi)=\left[r_{0}+i\left(r_{1} \xi-\frac{r_{-1}}{\xi}\right)\right] \frac{e^{-i \xi}}{i \eta \xi+1}
$$

where $\eta=T / \tau$ and $\xi$ has the meaning of dimensionless frequency. Note that regardless the used transformation of the frequency the Nyquist plots preserve their shape. In [11] it is shown that $\mathrm{MO}$ optimality for the more general class of plants (7) implies that

$$
1+2 \operatorname{Re} L(\xi) \rightarrow 0 \text { for } \xi \rightarrow 0 .
$$

This fact can be also seen in Fig. 1. Moreover, it was shown in [11] that the parameters $r_{k}$ are positive. Consequently, the open-loop Nyquist plot comes out from the point $(-0.5,-\infty)$ and it tends towards the right half-plane. It can be also seen in Fig. 1 that for the plant (1) $\operatorname{Re} L(\xi)$ is always increasing for $\xi \leqslant \xi_{u}$, where $\xi_{u}$ is the frequency when $\angle L(\xi)=-\pi$. Therefore

$$
L(\xi) \in\{z \mid \operatorname{Re} z>-0.5\}
$$


holds if $|L(\xi)| \leqslant 0.5$ for all $\xi \geqslant \xi_{u}$. In this case the closed-loop system is stable by the Nyquist criterion. However, if $\tau / T$ is sufficiently large, this condition is not fulfilled. In such cases $|L(\xi)|$ is increasing for $\xi \geqslant \xi^{\dagger}$, where $\xi^{\dagger}$ is some frequency larger than $\xi_{u}$.

Since from (20) it follows that

$$
\lim _{\xi \rightarrow \infty}|L(\xi)|=\lim _{\xi \rightarrow \infty} \sqrt{\frac{r_{0}^{2} \xi^{2}+r_{1}^{2} \xi^{4}+r_{-1}^{2}-2 r_{1} r_{-1} \xi^{2}}{\xi^{2}\left(1+\eta^{2} \xi^{2}\right)}}=\frac{r_{1}}{\eta}
$$

it is possible to conclude that the correction of the settings is needed if $r_{1} / \eta>0.5$. This observation has been used in [12], where the modified MO settings was proposed in the simple form

$$
r_{1}=\min \left\{r_{1}^{*}, 0.5 \eta\right\}
$$

where $r_{1}^{*}$ denotes the MO-optimal value of $r_{1}$ obtained by solving (13). The remaining coefficients $r_{0}, r_{-1}$ are obtained by solving the system of the first two equations in (13) for fixed $r_{1}$ :

$$
\left(\begin{array}{cc}
\eta+1 & -1 \\
3 \eta+1 & -3(2 \eta+1)
\end{array}\right)\left(\begin{array}{c}
r_{-1} \\
r_{0}
\end{array}\right)=\left(\begin{array}{c}
0.5 \\
-3 \eta^{2}-6 r_{1}(\eta+1)
\end{array}\right)
$$

i.e.

$$
\begin{aligned}
r_{-1} & =\left[-1.5(2 \eta+1)-3 \eta^{2}-6 r_{1}(\eta+1)\right] / D_{1}=-3\left[\eta^{2}+\eta+0.5+2 r_{1}(\eta+1)\right] / D_{1} \\
r_{0} & =\left[(\eta+1)\left(-3 \eta^{2}-6 r_{1}(\eta+1)\right)-0.5(3 \eta+1)\right] / D_{1}= \\
& =-3\left[\eta^{3}+\eta^{2}+\frac{1}{2} \eta+\frac{1}{6}+2 r_{1}(\eta+1)^{2}\right] / D_{1}
\end{aligned}
$$

where

$$
D_{1}=-3(\eta+1)(2 \eta+1)+3 \eta+1=-6 \eta^{2}-6 \eta-2
$$

The value of $\eta$, where just $r_{1} / \eta=0.5$, can be obtained by substitution into (15). The only real positive solution is $\eta_{d} \approx 0.1613$, which means that the correction of the settings is applied in the cases of $\tau>6.2 T$, which will indeed occur rarely in practice.

An advantage of this approach is its simplicity. On the other hand, the requirement that $|L(\xi)| \leqslant 0.5$ for $\xi \geqslant \xi_{u}$, which ensures (22), does not always give responses favorable from practical point of view. It can be seen in Fig. 4 that the obtained responses can be rather oscillatory, unlike the responses when $r_{1} / \eta<0.5$, which are usually well damped. This problem is caused by the open-loop resonance - note in Fig. 3 that even after the correction the magnitude response is not monotonic. This also means that the correction of the settings may be meaningful even for lower values of $\tau / T$. 


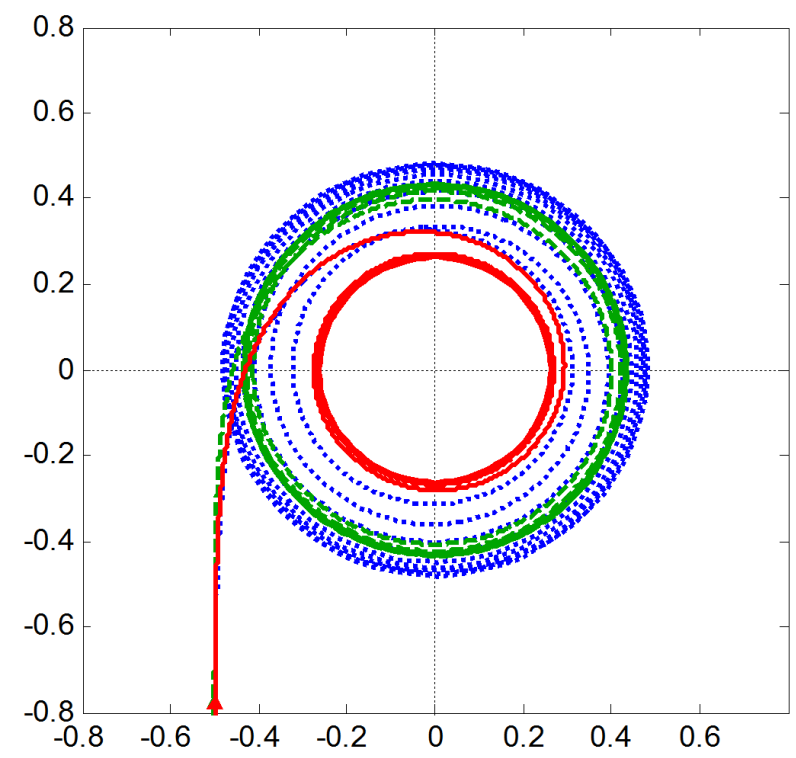

Figure 3. The open-loop Nyquist plots corresponding to the MO-optimal settings with simplified correction, where $\tau=1$ [sec] and $T=1$ [sec] (solid line), $T=0.2$ [sec] (dashed line) and $T=0.05$ [sec] (dotted line).

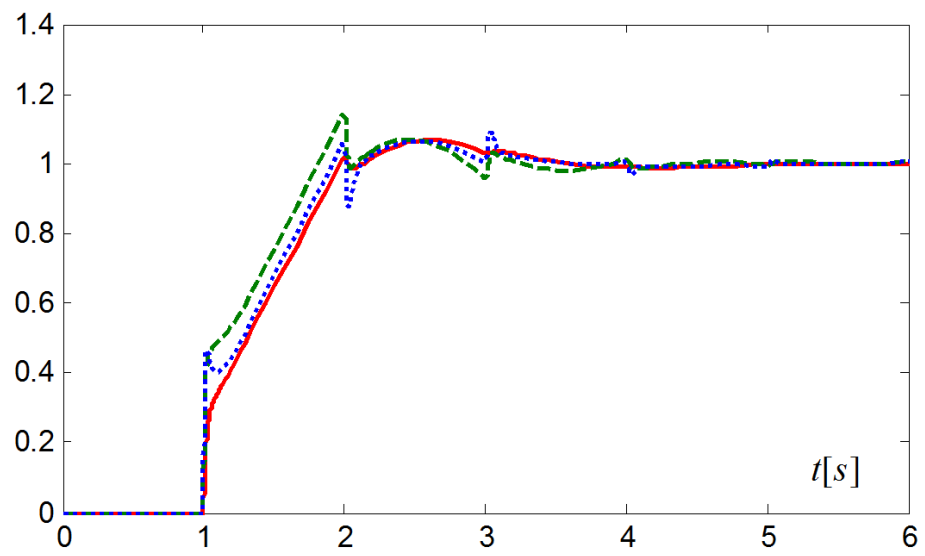

Figure 4. The step responses corresponding to the MO-optimal settings with simplified correction for plants (1), where $\tau=1$ [sec] and $T=1$ [sec] (solid line), $T=0.2$ [sec] (dashed line) and $T=0.05$ [sec] (dotted line).

\subsection{The enhanced method}

In [11] the correction of the MO settings for the class of systems (7) was proposed to keep $|L(\xi)|$ monotonic. The decreasing trend of $|L(\xi)|$ ensures (22) for $\xi \geqslant \xi_{u}$ and 
is natural with respect to practical requirements of well-damped closed-loop response. For the systems (7) the correction is unfortunately more complex and requires an iterative computation. However, we show that in the special case (1) it can be obtained in a simplified way. We can write the open-loop magnitude response as

$$
\begin{aligned}
|L(\xi)|^{2} & =\frac{r_{0}^{2}+\left(r_{1} \xi-r_{-1} / \xi\right)^{2}}{\eta^{2} \xi^{2}+1}=\frac{r_{1}^{2} \xi^{2}+\left(r_{0}^{2}-2 r_{1} r_{-1}\right)}{\eta^{2} \xi^{2}+1}+\frac{r_{-1}^{2}}{\xi^{2}\left(\eta^{2} \xi^{2}+1\right)}= \\
& =\left(r_{0}^{2}-2 r_{1} r_{-1}\right) \frac{\lambda \xi^{2}+1}{\eta^{2} \xi^{2}+1}+\frac{r_{-1}^{2}}{\xi^{2}\left(\eta^{2} \xi^{2}+1\right)}
\end{aligned}
$$

where

$$
\lambda=\frac{r_{1}^{2}}{r_{0}^{2}-2 r_{1} r_{-1}}=\left[\left(r_{0} / r_{1}\right)^{2}-2\left(r_{-1} / r_{1}\right)\right]^{-1} .
$$

It can be seen that it must be $r_{0}^{2}-2 r_{1} r_{-1}>0$ and consequently also $\lambda>0$ in the MO configuration, because otherwise the rate of decrease of $|L(\xi)|^{2}$ for $\xi \rightarrow 0$ would be larger than for the I-controller with $r_{0}=r_{1}=0$, which would mean that the settings $r_{0}=r_{1}=0$ was MO-optimal. The second term in (28) is always decreasing, while the first term in (28) is increasing if $\lambda>\eta^{2}$ and decreasing if $\lambda<\eta^{2}$. Note that if $r_{1} \rightarrow 0$, the magnitude response is always decreasing, because in this case

$$
|L(\xi)|^{2}=\frac{r_{0}^{2}+\left(r_{-1} / \xi\right)^{2}}{\eta^{2} \xi^{2}+1}=\frac{r_{0}^{2}}{\eta^{2} \xi^{2}+1}+\frac{r_{-1}^{2}}{\xi^{2}\left(\eta^{2} \xi^{2}+1\right)} .
$$

It is desirable to keep $\lambda$ as large as possible to ensure maximal possible bandwidth. This suggests the following modification of the MO settings:

If $\lambda>\eta^{2}$, put $\lambda=\eta^{2}$ and determine the corresponding $r_{1}$. The parameters $r_{0}, r_{-1}$ are obtained using (26). In the other cases the MO settings (14)-(16) are left unchanged.

The remaining problem is the determination of $r_{1}$ for $\lambda=\eta^{2}$. If we define $\rho=r_{1}^{-1}$, from equations (26) and (27) we can obtain

$$
\frac{r_{-1}}{r_{1}}=\gamma\left(0.5 c_{2} \rho+c_{1}\right), \quad \frac{r_{0}}{r_{1}}=\gamma\left(0.5 c_{3} \rho+c_{1}^{2}\right)
$$

where

$$
\begin{aligned}
c_{1} & =1+\eta \\
c_{2} & =\frac{1}{2}+\eta+\eta^{2} \\
c_{3} & =\frac{1}{6}+\frac{1}{2} \eta+\eta^{2}+\eta^{3}
\end{aligned}
$$

and

$$
\gamma=\left(c_{1} c_{2}-c_{3}\right)^{-1}=\left(\frac{1}{3}+\eta+\eta^{2}\right)^{-1}
$$


Substituting into (29) yields

$$
\lambda^{-1}(\rho)=\gamma^{2}\left(0.5 c_{3} \rho+c_{1}^{2}\right)^{2}-\gamma\left(c_{2} \rho+2 c_{1}\right)
$$

which for given $\lambda^{-1}=d$ is a quadratic equation for obtaining $\rho$, which can be rearranged as follows:

$$
0.25 \gamma^{2} c_{3}^{2} \rho^{2}+\left(\gamma^{2} c_{1}^{2} c_{3}-\gamma c_{2}\right) \rho+\left(\gamma^{2} c_{1}^{4}-2 \gamma c_{1}-d\right)=0 .
$$

The solution to (35) is obtained in the form

$$
\begin{aligned}
\rho & =\frac{\gamma c_{2}-\gamma^{2} c_{1}^{2} c_{3} \pm \sqrt{\left(\gamma c_{2}-\gamma^{2} c_{1}^{2} c_{3}\right)^{2}-\gamma^{2} c_{3}^{2}\left(\gamma^{2} c_{1}^{4}-2 \gamma c_{1}-d\right)}}{0.5 \gamma^{2} c_{3}^{2}}= \\
& =\frac{c_{2}-\gamma c_{1}^{2} c_{3} \pm \sqrt{c_{2}^{2}+2 \gamma\left(c_{1} c_{3}^{2}-c_{1}^{2} c_{2} c_{3}\right)+c_{3}^{2} d}}{0.5 \gamma c_{3}^{2}} \\
& =\frac{c_{2}-\gamma c_{1}^{2} c_{3} \pm \sqrt{c_{2}^{2}-2 c_{1} c_{3}+c_{3}^{2} d}}{0.5 \gamma c_{3}^{2}} .
\end{aligned}
$$

Further, we can write

$$
\lambda^{-1}(\rho)=\left(\frac{r_{0}}{r_{1}}\right)^{2}-2\left(\frac{r_{-1}}{r_{1}}\right)=\rho\left(r_{0}^{2} \rho-2 r_{-1}\right) .
$$

Since $\lambda^{-1} \rightarrow \infty$ for $\rho \rightarrow \infty, \lambda^{-1}(\rho)$ in (37) must be increasing in all the interval $\left[\rho_{2}, \infty\right)$, where $\rho_{2}=2 r_{-1} / r_{0}^{2}>0$, and it is the only interval where $\lambda^{-1}(\rho) \geqslant 0$, because $\rho \geqslant 0$. This means, for any given $d>\lambda_{M}^{-1}>0$, where $\lambda_{M}$ denotes the value of $\lambda$ corresponding to the MO-optimal settings, the equation (35) must have just one real positive solution. Moreover, due to increasing trend of $\lambda^{-1}(\rho)$, to larger $d$ there must correspond a larger value of $\rho$. Consequently, we can consider only ' + ' in place of ' \pm ' in (36).

The correction is to be applied only if $\eta<\eta_{\min }$, where $\eta_{\min }$ is such that

$$
\left(r_{0} / r_{1}\right)^{2}-2\left(r_{-1} / r_{1}\right)=\eta_{\min }^{-2}
$$

Substituting (14)-(17) into (38) gives $\eta_{\min }$ as the real solution to the equation

$$
\left(\frac{180 \eta^{5}+240 \eta^{4}+135 \eta^{3}+42 \eta^{2}+7 \eta}{60 \eta^{4}+60 \eta^{3}+27 \eta^{2}+7 \eta+1}\right)^{2}-30 \frac{12 \eta^{5}+12 \eta^{4}+5 \eta^{3}+\eta^{2}}{60 \eta^{4}+60 \eta^{3}+27 \eta^{2}+7 \eta+1}=1
$$

The equation (39) can be easily rearranged into an algebraic equation, which has only one positive real solution $\eta_{\min } \approx 0.2915$. Therefore, if $\eta<\eta_{\min }$, the value of $r_{1}$ is obtained from

$$
r_{1}=\frac{0.5 \gamma c_{3}^{2}}{c_{2}-\gamma c_{1}^{2} c_{3}+\sqrt{c_{2}^{2}-2 c_{1} c_{3}+\left(c_{3} / \eta\right)^{2}}}
$$


where $c_{k}$ and $\gamma$ are given by (32) and (33). The remaining parameters $r_{0}, r_{-1}$ then can be computed from (26), or using the expressions (31).

Fig. 5 shows the Nyquist plots corresponding to the same plants like in Fig. 1, but using this type of correction of the MO settings. Fig. 6 shows the corresponding step responses, which are more damped for low $\eta$ than the responses in Fig. 4.

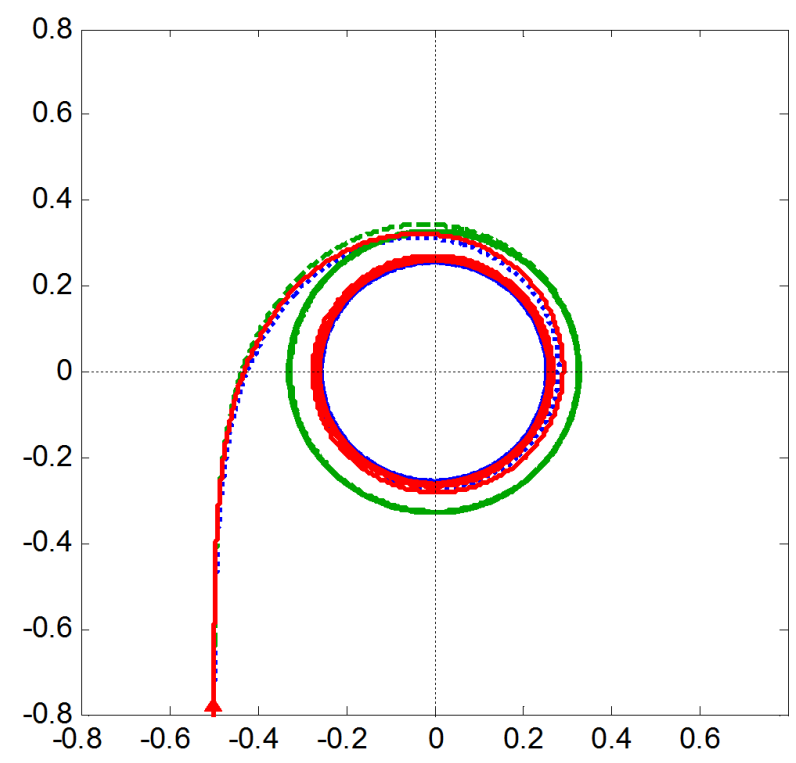

Figure 5. The open-loop Nyquist plots corresponding to the MO-optimal settings with enhanced correction, where $\tau=1$ [sec] and $T=1$ [sec] (solid line), $T=0.2$ [sec] (dashed line) and $T=0.05$ [sec] (dotted line).

It is clear from Fig. 6 that the final settings are only slightly dependent on $T$ and are similar to the settings for $T \rightarrow 0$ (represented approximately by the case $T=0.005$ [sec] in Fig.6). The fastest response with overshoot of about $10 \%$ was obtained for $T \approx 0.3 \tau$, for lower values of $T / \tau$ the response gets a bit slower due to the correction.

It can be easily seen from (40) or directly from (28) that for $T=0$ the proposed correction produces the settings such that $r_{1}=0$, which means that the corresponding controller is of type PI. Therefore, the closed-loop behavior for the proposed settings will be similar to the behavior in the case of the serial-type PID controller (4) where $T_{s d}=T$ is chosen to compensate the process lag and the remaining parameters $K_{c}$ and $T_{s I}$ are designed with respect to the MO criterion. The corresponding settings then are given by the simple tuning formulas (6) proposed in [13]. Therefore, it seems that for practical purposes the settings (14)-(17) with the enhanced correction can be replaced by the simple design rules (6). This also shows a rather surprising fact that that although these rules were developed only as sub-optimal with respect to the MO criterion, they produce settings very close to the optimal settings if the stability margin requirements are included into the design objective. 


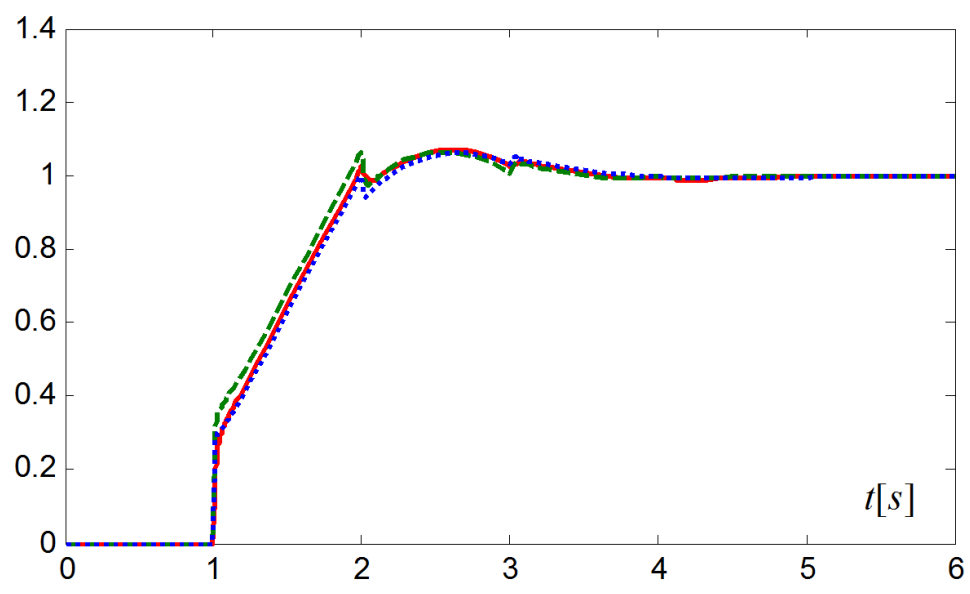

Figure 6. The step responses corresponding to the MO-optimal settings with enhanced correction for plants (1), where $\tau=1$ [sec] and $T=1$ [sec] (solid line), $T=0.2$ [sec] (dashed line) and $T=0.05$ [sec] (dotted line).

\section{Conclusions}

The modulus optimum criterion enables to design the PID controller for the FOPDT plant (1) analytically for any value of dead time $\tau>0$. For larger $\tau / T$, however, the stability margin gets reduced and even non-stable closed-loop behavior can be obtained. This problem can be resolved be a suitable modification of the settings, which keeps the open-loop Nyquist plot in the half-plane $\{z \mid \operatorname{Re} z \geqslant-0.5\}$. After this correction a sufficient stability margin and good robustness of the settings are guaranteed. We presented two methods of the correction, which try to preserve maximum of the performance of the original MO settings. For practical purposes especially the correction based on the requirement of monotonic open-loop magnitude seems to be advantageous. It was shown that for the FOPDT plant (1) this correction can be obtained in fully explicit form, which does not use any iterative computations, unlike the more general case (7), discussed in [11]. It was observed that the resulting settings with the correction give responses similar to the simple sub-optimal tuning rules (6) proposed in [13], which could be preferable for practical purposes.

\section{References}

[1] K.J. Åström and T. HÄGGLund: PID Controllers: Theory, Design, and Tuning. 2nd edition. Instrument Society of America. 1995. 
[2] K.J. Åström, H. Panagopoulos and T. HägGlund: Design of PI controllers based on non-convex optimization. Automatica, 34 (1998), 585-601.

[3] T.K. Kiong, W. Quing-Guo, H.CH. ChiECH and T.J. HÄGglund: Advances in PID Control. Springer Verlag. 1999.

[4] A. O'DwYER: Handbook of PI and PID Tuning Rules. Imperial College Press. 2003.

[5] J.G. ZIEGLER and N.B. NiCHOLS: Optimum settings for automatic controllers. Trans. of the ASME, 64 (1942), 759-768.

[6] G.H. Cohen and G.A. Coon: Theoretical consideration of retarded control. Trans. of the ASME, 75 (1953), 827-834.

[7] K.L. Chien, J.A. Hrones and J.B. Reswick: On the automatic control of generalized passive systems. Trans. of the ASME, 74, (1952), 175-185.

[8] A.M. Lopez, P.W. MURrill and C.L. SMITH: Controller tuning relationships based on integral performance criteria. Instrumentation Technology, 14(11), 1967.

[9] R.C. Oldenbourg and H. Sartorius: A uniform approach to the optimum adjustments of control loops. Trans. of the ASME, 76 (1954), 1265-1279.

[10] D. VRAncić, Y. Peng and S. STRMCniK: A new PID controller tuning method based on multiple integrations. Control Engineering Practice, 7 (1999), 623-633.

[11] J. CVEJN: The design of PID controller for non-oscillating time-delayed plants with guaranteed stability margin based on the modulus optimum criterion. $J$. of Process Control, 23 (2013), 570-584.

[12] J. CVEJN: PID control of FOPDT plants with dominant dead time - The frequency domain approach. Proc. of the 2013 Int. Conf. on Process Control, Strbske Pleso, Slovakia, (2013), 53-56.

[13] J. CVEJN: Sub-optimal PID controller settings for FOPDT systems with long dead time. J. of Process Control, 19 (2009), 1486-1495.

[14] D. VRANCIĆ, S. STRMCNIK and D. JURICIĆ: A magnitude optimum multiple integration tuning method for filtered PID controller. Automatica, 37 (2001), 14731479 . 